\section{Tranilast for early-stage diabetic nephropathy}

In 2002, Soma et al. showed that tranilast, an antifibrotic drug that suppresses collagen synthesis, slowed the progression of advanced diabetic nephropathy. These researchers have now investigated whether tranilast might also be useful in early-stage diabetic nephropathy.

The study included 20 outpatients with type 2 diabetes mellitus from a hospital in Japan. Inclusion criteria included a urinary albumin excretion of $30-1,000 \mathrm{mg} / \mathrm{g}$ creatinine in the first morning urine sample, a serum creatinine level of $\leq 1.2 \mathrm{mg} / \mathrm{dl}(106 \mu \mathrm{mol} / \mathrm{l})$ and no hematuria. All patients were taking an angiotensin-converting-enzyme inhibitor or an angiotensin receptor blocker. Ten patients were randomly assigned tranilast treatment (100 mg three times per day); the other patients received no additional therapy.

After 12 months, tranilast-treated patients showed significant decreases from baseline values in their excretion rates of both urinary albumin and urinary type IV collagen $(P=0.049$ and $P=0.02$, respectively). By contrast, excretion rates of urinary albumin and urinary type IV collagen tended to increase from baseline to 12 months in patients who did not receive tranilast. Blood pressure, serum creatinine and glycated hemoglobin levels remained stable during the study in both patient groups.

The authors conclude that tranilast might be a useful therapy for suppressing progression of early-stage diabetic nephropathy. A longerterm, prospective, controlled study is needed to further investigate the effects of this drug on diabetic nephropathy.

Original article Soma J et al. (2006) Effect of tranilast in early-stage diabetic nephropathy. Nephrol Dial Transplant 21: 2795-2799

\section{Novel treatment shows promise for type 1 primary hyperoxaluria}

Type 1 primary hyperoxaluria is a genetic disease caused by deficiency or absence of liver-specific alanine:glyoxylate aminotransferase, which leads to overproduction of endogenous oxalate. The disease is characterized by renal calculi, nephrocalcinosis and declining renal function, but treatment options are scarce. A recent study showed that Oxalobacter formigenes, a bacterium often present in the gut that is capable of degrading oxalic acid, might be able to degrade endogenously produced oxalate, thus lowering urinary oxalate excretion in patients with primary hyperoxaluria.

Two formulations of $O$. formigenes administered twice a day were tested. A frozen paste containing live cells was tested in five patients with normal renal function, three patients with end-stage renal disease (ESRD) and one patient who had undergone a liver-kidney transplant (IxOC-2 study); enteric-coated capsules containing freeze-dried live cells were tested in six patients with normal or stable renal function and one patient who had undergone a liver-kidney transplant (IxOC-3 study). Twenty-four-hour urine samples (or plasma samples in patients with ESRD) were analyzed weekly over 4 weeks of treatment. Patients achieving a decrease in their urinary oxalate excretion of $>20 \%$ at weeks 3 and 4 were classified as responders.

In the IxOC-2 study, three of the five patients with normal renal function responded to treatment, and plasma oxalate levels decreased in two of the three patients with ESRD. In the IxOC-3 study, four of the six patients with normal or stable renal function responded to treatment. The bacteria colonized the gut only transiently and no major adverse effects were reported.

Original article Hoppe B et al. (2006) Oxalobacter formigenes: a potential tool for the treatment of primary hyperoxaluria type 1. Kidney Int 70: 1305-1311

\section{Antiretroviral therapy retards progression of HIV-associated nephropathy}

The effect of antiretroviral therapy (ART) on kidney function in patients with HIV-associated nephropathy (HIVAN) is unclear. Atta et al. assessed the impact of ART on renal viability in a retrospective study of 36 patients with biopsy-proven HIVAN.

Data collected during the period 1995-2004 from patients referred to the Johns Hopkins renal clinic who did not require dialysis within 1 month of biopsy were reviewed. After 408 days of follow-up, renal survival was $52.7 \%$ in patients treated with ART $(n=26$, including those who received combination or monotherapy ART, or highly active ART) 